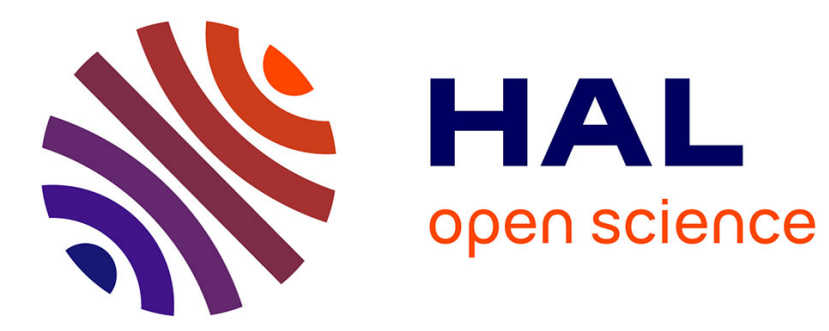

\title{
Les processus de sécurisation langagière des adultes : parcours sociaux et cursus d'apprentissage
}

\author{
Hervé Adami, Virginie André
}

\section{To cite this version:}

Hervé Adami, Virginie André. Les processus de sécurisation langagière des adultes: parcours sociaux et cursus d'apprentissage. Revue Française de Linguistique Appliquée, 2014, XIX (2), pp.71-83. hal01104924

\author{
HAL Id: hal-01104924 \\ https://hal.science/hal-01104924
}

Submitted on 13 Jan 2021

HAL is a multi-disciplinary open access archive for the deposit and dissemination of scientific research documents, whether they are published or not. The documents may come from teaching and research institutions in France or abroad, or from public or private research centers.
L'archive ouverte pluridisciplinaire HAL, est destinée au dépôt et à la diffusion de documents scientifiques de niveau recherche, publiés ou non, émanant des établissements d'enseignement et de recherche français ou étrangers, des laboratoires publics ou privés. 


\section{Les processus de sécurisation langagière des adultes : parcours sociaux et cursus d'apprentissage.}

\section{Hervé Adami, Virginie André ATILF (Université de Lorraine \& CNRS)}

Revue Française de Linguistique Appliquée, 2014/2, Vol XIX. p.71-83.

\section{Introduction}

Certains adultes sont en insécurité langagière, c'est-à-dire qu'ils possèdent des répertoires langagiers trop incomplets ou trop peu variés pour faire face avec efficacité aux situations de communication auxquelles ils participent, notamment celles inédites pour eux, hors de leurs espaces habituels d'interaction. Cette situation est due au fait que certains de ces adultes sont migrants, souvent faiblement ou non scolarisés dans leurs pays d'origine, et qu'ils doivent donc s'approprier la langue du pays d'accueil. Mais d'autres sont francophones et ceux-là sont en difficultés à l'écrit, voire à l'oral, avec leur « propre » langue.

Au-delà des différences apparentes entre natifs et non natifs, au-delà des débats sans fin autour des notions d'intégration, d'insertion ou d'inclusion, il s'agit ici de comprendre ce qu'est l'insécurité langagière, comment et pourquoi ces adultes se trouvent dans cette situation. Il s'agit également de savoir comment penser et construire des parcours de sécurisation langagière efficients dans le cadre des formations pour adultes.

\section{Intégration et insertion : le contexte social et économique.}

La crise, due à la mondialisation économique, sévit de façon plus ou moins violente, selon des cycles qui laissent parfois quelques répits, depuis les années quatre-vingt. Les taux de chômage «baissent » par intervalle quelques années mais restent à des niveaux qui entrainent 
des conséquences sociales toujours aussi graves. La France en particulier ne parvient pas à endiguer un chômage de masse qui mine littéralement la société. Au contraire, la désindustrialisation continue et les emplois perdus dans ce secteur ne sont que très partiellement compensés par des emplois de service ou des activités créant de fortes valeurs ajoutées comme l'ingénierie ou la recherche. Depuis les années quatre-vingt, l'économie française, mais également européenne, n'est plus capable d'occuper ses salariés les moins qualifiés qui sont souvent les moins bien pourvus en capital scolaire. Par ailleurs, entre 150000 et 200000 jeunes continuent à sortir tous les ans du système scolaire sans diplôme tandis que d'autres en possèdent mais avec une très faible valeur sur le marché du travail, notamment quand ils sont soumis à la concurrence d'autres jeunes diplômés prêts à accepter des emplois sous qualifiés. La conjonction de ces deux facteurs contribuent à créer une situation que les gouvernements successifs ne savent ou ne peuvent plus gérer.

Par ailleurs, dès les années quatre-vingt, avec la montée du chômage de masse, les acteurs du domaine de l'insertion font le constat que de nombreux salariés peinent à retrouver un emploi parce qu'ils éprouvent des difficultés en français. C'est le cas des travailleurs migrants et cela n'étonne personne puisqu'ils sont pour la plupart non natifs ou utilisent le français comme langue seconde ou étrangère. Mais les acteurs de l'insertion font un constat plus surprenant qui va remettre en cause quelques certitudes bien établies : des francophones natifs, scolarisés en France, éprouvent des difficultés très importantes à l'écrit. C'est la découverte, ou "l'invention» comme dirait Lahire (2005), de l'illettrisme. La crise a agi comme un révélateur de problèmes que le plein emploi industriel avait totalement masqué. En effet, dès lors que les emplois de main d'œuvre se raréfient et que la part langagière du travail (Boutet 2001) augmente, les difficultés linguistiques apparaissent et deviennent un véritable problème d'accès à l'emploi. La prise de conscience est réelle et les premiers constats sont suivis de mesures en termes de formation. A partir de la fin des années 
quatre-vingt, la « formation de base » (Leclercq 1999) va accueillir aussi bien les migrants que les francophones. C'est aussi à cette période, et dans ce contexte de crise aigüe, que le domaine de l'insertion va devenir un véritable champ professionnel. La formation des adultes, et en particulier la formation linguistique, se constitue à cette époque comme champs éducatifs de plus en plus structurés.

Migrants et francophones, chacun pour des raisons différentes mais avec les mêmes conséquences, sont donc concernés par l'insécurité langagière. Ce qui les rapproche également c'est qu'ils appartiennent, pour l'immense majorité d'entre eux, natifs et migrants, aux catégories populaires dont Schwartz propose une définition sociologique tout à fait claire et convaincante (2011). Dès le milieu des années soixante-dix, le linguiste Faïta fait le constat suivant :

On pourrait donc penser que l'ensemble des travailleurs français se trouvent dans une situation plus favorable, l'accès à la promotion sociale leur étant ouvert, dans le même temps qu'une discrimination de fait frappe la main d'œuvre étrangère. Or, et ce sont précisément les bilans et les comptes rendus de stage qui le révèlent, les adultes français de bas niveau éprouvent des difficultés d'expression qui, dans la mesure où elles affectent leurs relations socio-professionnelles, les placent dans une situation proche de celle de leurs homologues immigrés. (Faïta 1976, 28).

La remarque de Faïta n'est pas anodine parce qu'elle est faite une décennie avant que l'on «découvre » l'illettrisme, par un linguiste et, surtout, par un chercheur qui va consacrer une large partie de sa carrière à cette question des rapports entre langue et travail. Ce n'est donc pas la remarque d'un «touriste scientifique ».

Aujourd'hui, la crise frappe encore et toujours les salariés les moins bien dotés en capital scolaire (Bourdieu 1980) et notamment ceux qui sont le moins bien dotés en capital linguistique. Cependant, le constat que fait Faïta sans ambages au mitant des années soixante-dix est précisément contextualisé. La remarque de Faïta s'inscrit dans une démarche de recherche mais dans un contexte où il est question 
d'émancipation des travailleurs. Faïta fait ce constat ni pour s'apitoyer ni pour mépriser ; il écrit ainsi à la fin de son article :

Des hommes se voient condamnés à rester étrangers au monde où ils vivent, alors qu'ils contribuent à en créer les richesses. (Faïta 1976 : 30).

Ce qu'il constate donc sans édulcorer la réalité est bien un problème langagier mais il le fait avec en tête cette idée de l'émancipation. La « maitrise » de la langue, ou sa non maitrise en l'occurrence, est bien un problème politique. Aujourd'hui, le contexte intellectuel a changé et l'heure n'est plus à l'émancipation mais au relativisme culturel. Ces « difficultés d'expression » que constate Faïta ne sont plus analysées comme des freins à l'émancipation mais comme des variétés sociales stigmatisées par la culture linguistique dominante. Pour notre part, nous pensons, à la suite de Faïta, qu'il s'agit bien, hier et aujourd'hui, non d'un point de vue normatif et socio-centré sur des variétés délégitimées mais d'un véritable problème d'insécurité langagière. Et c'est le contact direct, ancien et régulier avec le terrain des adultes en insertion qui nous amène à formuler cette hypothèse, que des recherches en cours commencent à confirmer.

\section{La « maitrise » de la langue : débats et enjeux scientifiques et politiques.}

\subsection{Les origines du débat}

Une question importante se pose depuis les travaux fondateurs de Bernstein (1975) et Labov (1976): si l'on peut accepter le fait, scientifiquement démontré depuis longtemps, qu'il existe bien des variétés sociales de langue, peut-on établir une hiérarchie entre elles et peut-on affirmer qu'il existe des variétés supérieures à d'autres ? Si oui, quels sont les critères qui permettraient d'établir une telle supériorité ? Les travaux de Bernstein et Labov ont, entre autres, porté sur le langage 
des milieux populaires aux Etats-Unis et en Grande-Bretagne. Ils ont, chacun à leur manière, montré le lien existant entre les aspects sociaux et les aspects linguistiques et établi une corrélation entre l'appartenance sociale des locuteurs et la variété linguistique utilisée. Ils ont mené leurs recherches auprès de locuteurs appartenant aux milieux populaires et ont analysé à la fois les variétés qu'ils utilisaient mais également leurs façons d'interagir. Si Labov et Bernstein sont d'accord sur le fait que les variétés utilisées par les locuteurs des milieux populaires ne correspondent pas à la norme de l'anglais standard, et sur le fait que la complexité et la sophistication des variétés populaires est moindre que celle de la variété standard, ils ne sont en revanche pas d'accord sur un point fondamental : Bernstein conclut de ses recherches qu'il existe deux codes, l'un restreint et l'autre élaboré, et que le premier ne permet pas à ses locuteurs d'interagir efficacement dans toutes les situations de communication; Labov, au contraire, en conclut que les variétés utilisées par les locuteurs d'origine populaire ne sont certes pas conformes à la norme, mais qu'elles sont parfaitement à même de répondre aux besoins langagiers des locuteurs, dans des types d'interactions très différents, et d'aborder des thèmes qui nécessitent un certain niveau d'abstraction. Selon Labov, les variétés langagières des locuteurs issus des milieux populaires souffrent d'abord d'un problème de reconnaissance et de légitimité sociale mais qu'elles ne sont pas intrinsèquement inférieures aux variétés standard. Nous retrouvons ici l'opposition bien connue variétés dominées/variétés dominantes que reprend notamment Bourdieu (1982). Les conclusions de Bernstein ont été reprises dans le domaine scolaire pour appuyer la thèse dite du «handicap linguistique » qui permet, à propos de l'échec scolaire, de dédouaner l'école de ses responsabilités en accréditant l'idée que le problème ne vient pas de l'institution scolaire et de ses acteurs mais d'un problème sociolangagier qui pénalise les enfants d'origine populaire. Il convient cependant de préciser que cette notion de handicap linguistique n'a pas été avancée par Bernstein lui-même. 
Le débat entre Labov et Bernstein est lui-même contextualisé. Labov est américain et lutte contre les théories pseudo-scientifiques du moment qui cherchent à expliquer la situation sociale désastreuse des noirs américains en montrant que leurs résultats aux tests d'intelligence sont inférieurs à ceux des blancs. Labov prend donc le parti de démontrer, de son point de vue de linguiste, que les variétés linguistiques utilisées dans les ghettos noirs ne sont pas inférieures à l'anglais standard mais qu'elles sont tout aussi efficaces et à même d'exprimer les pensées les plus complexes. La position de Labov est d'abord antiraciste. Bernstein, lui, est anglais et il vit dans une société où la stratification de classe est très marquée et où le prolétariat peine à sortir de sa condition, notamment du fait d'un faible niveau d'éducation. L'idée sous-jacente de Bernstein est de montrer les difficultés linguistiques pour y remédier.

\subsection{Vers l'appropriation collective des moyens de production symbolique}

Nous ne voulons pas adopter ici la position bien commode qui consiste à dire que la vérité se trouverait quelque part entre les deux mais me positionner par rapport à l'un et à l'autre auteur. Comme Labov et Bourdieu, nous pensons effectivement que le poids des variétés linguistiques dominantes, académiques, scolaires, littéraires voire médiatiques aujourd'hui, est déterminant et qu'elles servent de parangon, non d'abord par leur « qualité » relatives, mais parce qu'elles sont les variétés construites par et pour les classes dominantes. Cependant, nous pensons avec Bernstein, que les variétés utilisées par ces locuteurs ne leur permettent pas d'interagir efficacement dans toutes les situations de communication dans lesquelles ils sont engagés au quotidien. La description que fait Bernstein du code restreint nous semble bien adaptée à ce que l'on peut empiriquement constater sur le terrain, au contact des adultes en insertion. 
On sait bien depuis Bourdieu (1982) et tous les travaux en sociolinguistique depuis plusieurs décennies, que les variétés linguistiques ne se valent pas dans «l'économie des échanges linguistiques ». Les dichotomies classiques langue dominante/langue dominée, variété légitime/variété minorée ou bien norme/contrenorme sont à notre avis à dépasser parce qu'elles ne rendent plus compte de la complexité de la réalité. L'enjeu aujourd'hui est de comprendre, d'un point de vue langagier, ce qui permet, ou au contraire ce qui empêche, l'accès universel aux ressources symboliques de toutes natures. L'accumulation, la diffusion et la transmission des connaissances, et l'accès à ce savoir, nécessitent la maîtrise de formes langagières et discursives spécifiques et cette appropriation passe par un apprentissage. Le relativisme culturel radical a pour conséquence le maintien du statu quo socio-linguistique. La «langue dominante» d'aujourd'hui c'est aussi la langue des médias, de l'école, de l'université et de la recherche, de la technocratie, etc. Ainsi, dire qu'il n'y a pas d'autre problème que celui d'une légitimité ou d'une reconnaissance, c'est laisser dans une situation de dominés ceux qui n'ont pas accès à l'une ou l'autre des variétés dominantes.

A cet égard, nous pensons nécessaire de relire deux discours que nous croyons fondateurs de la politique linguistique de la France moderne et qui marquent par leur modernité. Il s'agit des interventions de Bertrand Barère et Henri Grégoire à la Convention en 1794. Ces deux révolutionnaires intransigeants, qui entendaient aller jusqu'au bout de la rupture avec l'Ancien Régime, sont bien plus pragmatiques en matière linguistique : s'ils veulent éradiquer les patois et imposer le français, ce n'est pas par une volonté d'uniformisation ou de nivellement, mais parce que le français est à leur yeux à un outil performant d'émancipation politique et que sa maîtrise permet l'accès au savoir et facilite la communication entre les membres de la communauté de citoyens qu'est en train de devenir la Nation républicaine. Ecoutons Barère (De Certeau, Julia \& Revel 1975, 327329) : «Citoyens, la langue d'un peuple libre doit être une et la même 
pour tous. (...) Donnons donc aux citoyens l'instrument de la pensée publique ; l'agent le plus sûr de la révolution, le même langage. » $\mathrm{Ou}$ bien encore: «Dans la démocratie, au contraire, la surveillance du gouvernement est confiée à chaque citoyen ; pour le surveiller il faut le connaitre, il faut surtout en connaitre la langue ». L'enjeu véritablement révolutionnaire n'est pas de détruire la langue « dominante » mais de se l'approprier : « Nos ennemis avaient fait de la langue française la langue des cours; ils l'avaient avilie. C'est à nous d'en faire la langue des peuples, et elle sera honorée». La conséquence de cette nouvelle politique linguistique c'est, comme le proposent les deux révolutionnaires, la création d'un service national, public, laïque et gratuit d'instruction ouvert à tous, y compris aux femmes, qui ne sera mis en place qu'un petit siècle plus tard.

Dans la grande enquête dirigée par Bourdieu sur cet univers populaire si méconnu (Bourdieu 1993), sont plusieurs fois pointés des problèmes d'insécurité langagière avec leurs conséquences humaines et sociales. Dans cet ouvrage, Accardo évoque le cas de Sébastien, un jeune lycéen issu d'une famille populaire qui dit, parlant de son père : « Il ne manque aucun conseil de parents d'élèves et multiplie les entrevues avec les professeurs bien que celles-ci, précise Sébastien, soient pour lui, « qui ne parle pas très bien », autant d'occasions de "prendre des gifles du milieu enseignant » ( (Bourdieu 1993, 1107). C'est le même problème que relatent Pialoux et Beaud à propos des ouvriers de Sochaux : « Les militants qui, à l'usine, ont été confrontés à des situations de type scolaire, d'intimidation symbolique (les négociations avec la direction du personnel, les discussions avec les représentants de l'Etat, les réunions de Comité d'Entreprise, etc.) ont «mesuré » l'importance qu'il y a à bien maîtriser le langage, l'arme que constituent les différentes formes de l'aisance culturelle, et inversement ont pu mesurer le prix qu'ils ont parfois payé - en humiliations, intimidations, impuissance, ou rage rentrée, notamment dans les circonstances « officielles » pour leur « inculture » (relative), et l'effort qu'ils ont dû faire pour se remettre « dans le bain », par exemple face aux lectures liées à la vie syndicale (le 
droit du travail, les textes de loi, la compréhension des mécanismes économiques de base et des statistiques, etc.). Aujourd'hui, ils savent bien que le «coup de gueule » contre le chef d'équipe, les stratégies de « retournement symbolique » ne marchent plus comme avant.» (Bourdieu 1993, 511-512).

Aujourd'hui, l'enjeu est toujours le même: l'accès véritablement démocratique à l'appropriation de cette langue/instrument indispensable qu'est le français «standard » ou les français dominants, c'est-à-dire ceux de l'école, des médias, de la recherche, de la littérature, de la chanson, des métiers, etc. Il s'agirait, pour paraphraser un célèbre mot d'ordre, d'aller vers l'appropriation collective des moyens de production symbolique.

\section{Les rapports « populaires » au langage.}

S'agissant des personnes à faible capital scolaire engagées dans des dispositifs d'insertion ou d'intégration, la question de leurs rapports spécifiques au langage se pose. Nous avons évoqué Bernstein et Labov mais le problème a été abordé de façon plus générale sur les « cultures » populaires ou sur les rapports «populaires » au savoir et à la culture dominante. L'approche scientifique de ces rapports ellemême pose problème. Grignon et Passeron (1989) ont bien montré que ces approches étaient constamment en tension entre deux pôles : ce qu'ils appellent le «misérabilisme » et le «populisme ». Les approches « misérabilistes » selon eux tendent à aborder la question des rapports populaires à la culture dominante par une sorte de position de surplomb qui prendrait la langue ou la culture dominante comme référence pour constater, relever, déplorer les manques, les déficits des pratiques populaires de la culture ou de la langue. Les approches «populistes» au contraire abordent la question sous l'angle du relativisme et entendent analyser les pratiques culturelles ou langagières populaire en soi, sans prendre de référence dans les 
pratiques dominantes. « Misérabilisme » et «populisme » sont bien des pôles dont se rapprochent plus ou moins certaines approches scientifiques : il ne s'agit pas de classer ces approches dans ces deux catégories mais de comprendre où elles se situent sur le continuum qui va d'un pôle à l'autre. Des travaux importants ont été menés par Hoggart (1970) et De Certeau (1990) qui montrent que les « cultures » populaires ne se définissent pas par rapport aux pratiques dominantes mais qu'elles possèdent leurs propres références, qu'elles existent par elles-mêmes, aux côtés voire contre les pratiques dominantes. L'analyse des pratiques langagières des classes populaires se heurte toujours au même écueil, que les auteurs tentent d'analyser : «l'ethnocentrisme de classe » comme l'appelle Grignon dans sa préface du livre de Hoggart (op cit). En effet, l'analyse des pratiques langagières des classes populaires est menée par ceux qui précisément possèdent le capital culturel à partir duquel ils construisent leurs catégories d'analyse. Hoggart par exemple, et c'est en cela que ses travaux sont fondateurs, construit son analyse «de l'intérieur» en analysant les pratiques culturelles des classes populaires de son point de vue de «transfuge», étant lui-même issu de ce groupe social. D’autres comme Verret (1996) tente d'analyser ces pratiques par une approche descriptive minutieuse des faits et gestes du quotidien. C'est le cas également de Sansot (1991), sur un mode moins distancié et plus narratif, en prenant lui aussi appui sur son appartenance aux catégories populaires. Ces travaux ne sont pas centrés sur les rapports au langage mais il en est toujours question. Dans l'univers des pratiques symboliques et sociales, le langage occupe en effet une place centrale et cette question revient sans cesse dans ces travaux plutôt sociologiques ou ethnographiques. Bernard Lahire, sociologue lui-aussi, a en revanche abordé le problème directement. $\mathrm{A}$ partir de ses premiers travaux sur les difficultés scolaires à l'écrit des enfants d'origine populaires (1994), il étend son analyse aux adultes (Lahire 1993a ; 1998). Il est lui-même un «rescapé » comme il l'écrit (1993b), c'est-à-dire d'origine populaire, et cette précision n'est pas anecdotique dans le débat sur «l'ethnocentrisme de classe » que nous 
venons d'évoquer. Son analyse sur les rapports à l'écrit et au langage des adultes des classes populaires est particulièrement pertinente et permet d'éclairer les pratiques, les comportements ou les réactions de ces adultes quand ils sont en formation par exemple. Lahire (1993a) écrit par exemple :

L'invariant des différents modes d'appropriation des textes (journaux, revues, livres divers) mis en œuvre par ceux-ci, c'est la volonté objective d'ancrage des textes dans une autre réalité que la seule réalité textuelle: dans une configuration pratique, dans un espace connu, vécu, dans les cadres, les schémas d'une expérience passée ou présente ou dans le réel.

Ce que montre Lahire, c'est que les adultes à faible capital scolaire ne conçoivent pas le langage, écrit ou oral, comme un objet qui puisse être mis à distance et que l'on peut analyser. Lahire (1993b) écrit encore :

Pour eux la fonction du langage réside dans son efficace pratique au sein de situations où il est ignoré comme tel. Ce qui compte pour eux, ce sont les situations d'énonciation où le langage agit et où l'on agit par le langage (...) et non le langage en tant que tel (per se) détaché des situations de production du sens dans les multiples échanges verbaux.

C'est ce que confirment nos propres travaux avec des ouvriers en formation (Adami 2008). Cet aspect des rapports «populaires» au langage est selon déterminant.

\section{Les insécurités langagières}

\subsection{Définition}

Nous définissons l'insécurité langagière comme la difficulté pour un locuteur/scripteur de gérer de façon efficace les interactions verbales dans lesquelles il est engagé, d'un point de vue linguistique, interactionnel, pragmatique et social. L'insécurité langagière n'est pas mesurable dans l'absolu mais son degré est variable en fonction des situations de communication dans lesquelles l'interactant est engagé, de 
ses interlocuteurs, des thèmes abordés, de l'objectif de communication et, bien sûr, de la langue ou de la variété de langue utilisée au cours de cette interaction (Hymes 1972). Concernant la capacité de gestion des interactions, elle dépend d'un certain nombre de facteurs qui ont été depuis longtemps décrits par les travaux concernant l'approche communicative en didactique des langues. Le locuteur est considéré comme plus ou moins compétent selon qu'il maîtrise les aspects linguistiques, discursifs, pragmatiques ou socioculturels de la communication.

Dans le cas d'une interaction exolingue (Alber \& Py 1985 ; Alber \& Py 1986 ; De Pietro 1988), l'insécurité langagière dépend très fortement des aspects linguistiques puisque, dans ce cas, la langue utilisée n'est pas la langue première d'au moins l'un des interlocuteurs. L'insécurité langagière des migrants non francophones est donc un fait qui relève du problème général de la compétence de communication (Hymes 1984).

En revanche, l'insécurité langagière des locuteurs engagés dans une interaction endolingue est beaucoup plus difficile à cerner, à comprendre et même à admettre. Et pourtant, si l'on reprend point par point les composantes de la compétence de communication, dont Moirand (1982) a proposé la synthèse par exemple, et qu'on les utilise pour l'analyse d'interactions endolingues engageant des individus en insertion et/ou faiblement scolarisés, on peut dégager un certain nombre d'éléments précis qui peuvent nous permettre de définir la nature de cette insécurité et d'en mesurer l'étendue. Les éléments décrits par Bernstein (1975) pour définir ce qu'il entend par «code restreint » et « code élaboré » sont des bases intéressantes mais il faut y ajouter les aspects interactionnels, pragmatiques et sociaux ou sociolinguistiques qui peuvent parfois compenser l'insécurité spécifiquement linguistique. En tenant compte de ces aspects, on évite également de focaliser sur les seuls aspects codiques qui sont parfois trop réducteurs. Interagir de façon efficace, c'est comprendre et se faire comprendre, au moins sur l'essentiel ; c'est être capable d'intervenir dans une interaction en utilisant toutes les ressources à sa disposition 
pour co-construire le discours et le sens, au-delà des aspects purement linguistiques ; c'est savoir se décentrer en faisant les bonnes hypothèses sur ce que sait ou ne sait pas son interlocuteur ; c'est savoir utiliser les implicites de tous ordres à bon escient et de façon consciente ; c'est être capable de construire un message cohérent en utilisant des formes linguistiques (lexicales, morphologiques, syntaxiques, discursives) qui soient à même de faciliter l'intercompréhension sans pour autant respecter toutes les normes ou les sur-normes académiques.

L'insécurité langagière concerne aussi bien l'oral que l'écrit. A l'oral, il s'agit de l'ensemble des interactions interpersonnelles, transactionnelles, professionnelles dans lesquelles sont engagés les interlocuteurs et où sont constatés des problèmes de communication et d'intercompréhension. A l'écrit, l'insécurité langagière est un problème qui relève de l'analphabétisme, de l'analphabétisme fonctionnel ou de l'illettrisme (Besse 1995, Leclercq 1999). Ces questions relèvent toutes de l'approche théorique générale dont le concept de littéracie rend compte. La littéracie concerne à la fois la description et l'analyse de l'écrit dans ses dimensions sémiotique, anthropologique, historique, sociale et cognitives, à la suite des travaux fondateurs de Goody (1979, 1986, 1994, 2007), mais également l'analyse et la description des processus d'apprentissage et d'acquisition de la lecture et de l'écriture, c'est-à-dire d'appropriation de l'écrit, d'acculturation à l'écrit, par des enfants ou des adultes, natifs ou non.

\subsection{Points de vue d'observation: la formation d'adultes, l'insertion, le travail}

Les insécurités langagières des adultes natifs et non natifs en situation de formation linguistique, d'insertion professionnelle ou en situation de travail sont généralement méconnues, mal identifiées ou encore négligées, ces trois constats étant fortement liés les uns aux autres. 
Cependant, comme nous l'avons précisé plus haut, ces insécurités langagières peuvent être à l'origine de problèmes d'intégration sociale et/ou économique voire de problèmes de sécurisation dans l'emploi. Lors d'un séminaire du réseau Langage, Travail et Formation ${ }^{1}$, nous avons analysé les termes utilisés par les commanditaires de formation et par les formateurs eux-mêmes pour décrire le public en insécurité langagière, ainsi que de leurs objectifs de formation (LTF 2012). Cette analyse montre que, pour eux, les contours de cette notion sont flous.

En effet, de la part des commanditaires, nous trouvons des formulations du type « ils ont du mal à se faire comprendre, l'objectif de la formation est donc qu'ils arrivent à se faire comprendre ", "ils sont mauvais ou moyens » (demande de formation du secteur Bâtiment Travaux Publics), «ils doivent comprendre un texte», «améliorer leur expression écrite » (dispositif Compétences Clés ${ }^{2}$ ), «ils doivent savoir lire-écrire-compter » ou encore « acquérir les compétences de base sur le plan linguistique » (demande de formation du secteur de l'hôtellerie). Ainsi, les acteurs sociaux ou professionnels confrontés à des personnes en insécurité langagière ne savent pas bien la décrire et la mesurer. Ils ont bien l'intuition qu'il y a un problème langagier lorsqu'ils constatent les difficultés que rencontrent certains salariés à être opérationnels en situation de travail, à trouver un emploi ou encore à s'intégrer à la société dans laquelle ils vivent mais ils ne réussissent pas à l'identifier, à le nommer précisément et à trouver des solutions en termes d'objectifs de formation.

Du coté des formateurs, les contours de l'insécurité langagière sont également flous. Dans le cadre d'une recherche-action visant à analyser les pratiques professionnelles des formateurs en Français Langue d'Intégration (Adami, André, à paraître), une enquête que nous avons réalisée dans le Grand-Est de la France (Lorraine, Franche-Comté, Alsace) montre que les formateurs, quels que soient leur formation, leur âge, leurs années d'expériences, l'organisme de formation dans lequel

\footnotetext{
${ }^{1}$ https://apps.atilf.fr/reseaultf/

${ }^{2}$ Il s'agit d'une formation aux « compétences fondamentales », notamment pour les salariés et les demandeurs d'emploi (http://www.emploi.gouv.fr/dispositif/competences-cles).
} 
ils travaillent, ont tous des difficultés pour définir précisément les insécurités langagières de leurs apprenants ou stagiaires. Ils expliquent également éprouver des difficultés à appréhender de façon efficace ces insécurités, c'est-à-dire à tenter d'y remédier en séance de formation. Cette enquête auprès de formateurs confirme également un constat que nous avions pu faire par ailleurs (LTF 2012). En effet, tous les formateurs de notre enquête soulignent les difficultés qu'ont les personnes en insécurités langagières pour «apprendre». Plus précisément, certains formateurs expliquent que les problèmes de compréhension orale ou écrite sont liés à une incapacité d'abstraction. Nous pensons notamment à une formatrice qui explique que l'activité « choisir un plat dans un menu » proposée à un apprenant a posé problème parce que le menu ne ressemblait pas à celui que connaissait l'apprenant. Ainsi, les problèmes d'abstraction des personnes en insécurité langagière renforcent les difficultés d'une formation visant justement des processus de sécurisation langagière.

En situation de travail, l'évolution des métiers accordant une place de plus en plus importante à la part langagière du travail (Boutet 2001, André 2009), le repérage, en situation de travail ou en situation d'évaluation ou de positionnement linguistique, des insécurités langagières des salariés est grandissant. En effet, de plus en plus d'entreprises ou de branches professionnelles repèrent des salariés qui sont désormais en difficulté pour effectuer leur travail. Les besoins langagiers des salariés étant sans cesse en augmentation, notamment avec l'apparition de nombreuses certifications, concernant la sécurité au travail, par exemple, les besoins de formation des personnes en insécurité langagières ne cessent également d'augmenter. Les enjeux de formation sont importants, ils permettent aux salariés de conserver leur emploi ainsi que de participer pleinement à leur activité de travail. 


\subsection{L'insécurité à l'écrit}

Le simple fait selon nous que des personnes soient en insécurité à l'écrit suffit pour affirmer qu'ils sont en insécurité langagière, même si la communication à l'oral ne pose pas de problème particulier. En effet, sauf à considérer que l'écrit ne fait pas partie des compétences langagières, c'est un aspect majeur de socialisation, de communication, d'acquisition et de structuration des connaissances. Les difficultés à l'écrit ont bien sûr des conséquences sur les parcours scolaires, et donc sur l'entrée dans la vie professionnelle, mais également sur le parcours et les situations professionnelles elles-mêmes, sur l'aide aux enfants, sur la vie quotidienne, etc. Les difficultés à l'écrit peuvent entrainer une déscolarisation et une désocialisation.

Au-delà de ces aspects, l'écrit et l'oral sont indissociables : d'une part parce que l'écrit procède de l'oral dans les systèmes d'écriture alphabétique et que l'un et l'autre sont indissolublement et contradictoirement liés; d'autre part, parce que les compétences acquises à l'écrit influent sur celles de l'oral, et inversement. On le savait déjà pour les deux compétences de l'écrit, la lecture et l'écriture : les travaux de Ferreiro (2001) notamment, ont montré que le fait d'écrire favorise l'apprentissage de la lecture parce que cela développe certaines compétences métalinguistiques. Les travaux fondateurs de Laurence Lentin (Canut \& Vertalier 2008) ont également montré que les rapports à l'oral des enfants déterminaient de façon décisive leurs rapports à l'écrit : l'entrée dans l'univers de l'écrit passe par l'entrée dans l'univers du langage et notamment par les interactions langagières avec les adultes. L'oral et l'écrit ne sont pas des univers sémiotiques, communicatifs ou langagiers étanches : au contraire, les passages de l'un à l'autre, d'une compétence à l'autre, sont le fait quotidien des locuteurs/scripteurs/interlocuteurs/lecteurs.

Les adultes en insécurité scripturale sont donc, sans même évoquer le problème de l'oral, en insécurité langagière. Si l'on se base sur ces aptitudes pour construire un parcours de formation avec des 
analphabètes par exemple, la moitié de ces compétences n'existent tout simplement pas et elles sont très limitées à l'écrit avec des adultes faiblement scolarisés. L'insécurité scripturale est donc bien une forme d'insécurité langagière d'une part parce que l'écrit est une compétence langagière à part entière et, d'autre part, parce que sans ces compétences scripturales, le développement de celles de l'oral est obéré.

\subsection{L'insécurité à l'oral}

Les phénomènes d'insécurité langagière sont décrits et assez bien connus dans le cadre de l'analyse des interactions exolingues et/ou chez les migrants qui acquièrent la langue du pays d'accueil. En ce qui concerne les natifs interagissant dans leur langue première, les études sont moins nombreuses et les acquis scientifiques sont beaucoup moins évidents. En ce qui concerne les compétences des natifs à l'oral en revanche, les avancées scientifiques sont très relatives et sont par ailleurs parasitées par un débat idéologique et scientifique intense. Lapoutre par exemple (2001), fréquemment cité à l'appui de certaines thèses «populistes », au sens qu'en donne Grignon et Passeron (1989), menant une enquête sur les jeunes de banlieues, aborde la question du langage et il en conclut qu'il n'existe pas chez eux de « déficit » langagier en prenant pour preuve l'habileté de ces jeunes dans les joutes verbales ou dans l'expression artistique musicale. Or, outre le fait que ces jeunes ne représentent qu'une petite partie des classes populaires, et ne sont donc pas sociologiquement représentatifs, l'enquête de Lapoutre ne porte que sur les jeunes qu'il a rencontrés et qui s'adonnent à ces exercices langagiers en «oubliant» tous les autres. L'enquête de Lapoutre est l'arbre «artistique» qui cache la forêt de l'insécurité langagière. La «preuve » par le slam, le rap, le cinéma ou les joutes verbales et une sorte de tarte à la crème argumentative très utilisée dans le débat public par ceux qui tentent de faire croire qu'il n'y pas de problème d'expression mais qu'il s'agirait uniquement d'un point 
normatif socio-centré. C'est typiquement la position «populiste» décrite selon Grignon et Passeron (1989).

Cependant, quelles que soient les positions des uns et des autres, les recherches portent toujours sur l'expression orale des enquêtés dont on analyse les discours produits en situation plus ou moins expérimentale. Aucune enquête ne porte sur la compréhension orale des natifs dans leur langue.

Afin de tenter de pallier ce manque, nous avons commencé une enquête préliminaire visant à évaluer le degré éventuel d'insécurité langagière en compréhension orale des adultes natifs en situation d'insertion face à un discours courant en français. Pour mener à bien cette enquête, nous avons choisi un public d'adultes et de jeunes adultes en insertion, suivant des formations dans le cadre du dispositif Compétences Clés ${ }^{3}$. Notre échantillon comporte des salariés et des demandeurs d'emploi, de 19 à 47 ans, faiblement scolarisés, avec peu d'expérience professionnelle. Nous avons choisi un document sonore professionnel, non technique, susceptible d'être entendu par l'ensemble de nos informateurs. Il s'agit d'un document informatif général sur les consignes de sécurité et plus précisément sur la prévention des risques de surdité liée à une exposition prolongée au bruit. Les résultats de cette enquête préliminaire montrent que, même si les degrés de compréhension varient en fonction des individus et notamment en fonction de leur connaissance du thème, nous pouvons qualifier la compréhension du document comme une compréhension de surface. Nous constatons que chaque personne est capable de restituer environ 5\% du document sonore. Certains restituent des mots clés entendus au fil de l'écoute, d'autres prennent appui sur des indices, parfois hasardeux, pour évoquer leur propre expérience du bruit en situation de travail voire une expérience vécue sans véritable lien avec la thématique du document diffusé. De façon générale, la compréhension reste globale, aucun détail, pourtant nombreux dans le document, n'est

\footnotetext{
${ }^{3}$ Voir supra, note 1.
} 
restitué. En outre, les difficultés liées à l'évaluation de la compréhension orale apparaissent avec les difficultés d'expression des éléments potentiellement compris. Les premiers résultats de cette enquête mettent au jour de véritables problèmes de compréhension orale, par conséquent des traces d'insécurité langagière à l'oral, alors que cette dimension reste un impensé de la recherche, contrairement à l'illettrisme comme nous l'avons mentionné précédemment ${ }^{4}$.

Ainsi, au-delà des positions idéologiques, il existe bien des difficultés de communication à l'oral avec des locuteurs natifs en insertion. Ces difficultés sont très variables et leur fréquence, ainsi que leur « gravité », correspondent le plus souvent au niveau de scolarisation. En effet, plus le niveau scolaire des locuteurs est faible et plus les difficultés de communication sont fréquentes et importantes. Langbach (2014 et dans ce numéro) montre bien, en analysant un important corpus d'interactions verbales entre des demandeurs d'emploi et des conseillers en insertion, quelles sont les difficultés de communication à l'oral de ces adultes, notamment au niveau de la co-construction du discours et de la gestion de l'interaction.

Quels sont donc les grands traits de la conduite des interactions par ces adultes en insécurité langagière ? Sans reprendre tels quels les travaux et les résultats de Bernstein, il nous semble possible, et surtout fructueux, de revenir sur les problèmes qu'il soulève en s'appuyant sur les avancées décisives dans le domaine de l'analyse des interactions verbales et notamment sur la co-construction du sens.

$\mathrm{Au}$ cours des interactions avec des adultes en insécurité langagière et/ou sociale, on peut constater une série de traits interactionnels récurrents dont la fréquence nous semble spécifique aux échanges avec les personnes en insertion, natives et faiblement scolarisées.

1) Nombre important d'hétéro-reformulations effectuées par le locuteur « expert » dans un but d'intercompréhension.

\footnotetext{
${ }^{4}$ Il est d'ailleurs intéressant de noter que l'insécurité langagière à l'écrit porte un nom, celui d'illettrisme, qui n'a pas d'équivalent pour l'oral.
} 
2) Interventions fréquentes du locuteur « expert» sous forme de questions fermées qui anticipe les propos de son interlocuteur qui n'a plus qu'à approuver ou à désapprouver.

3) Présence de nombreux implicites dans le discours du locuteur en insécurité renvoyant à la situation d'énonciation, à son histoire conversationnelle avec d'autres interlocuteurs, à des référents que l'interlocuteur en présence ne peut comprendre faute de savoir partagé, etc.

4) Présence dans le discours du locuteur en insécurité de reprise anaphoriques aléatoires.

5) Discours «en spirale», qui se répète, par des reprises paraphrastiques entre autres.

6) Présence de nombreux pantonymes.

7) Interventions très souvent interrompues en cours de réalisation par le locuteur en insécurité, hésitations mais également ruptures thématiques, nombreux régulateurs marquant l'hésitation (bon, ben, alors, hein, euh, etc.).

Ces traits ne sont pas spécifiques aux locuteurs en insécurité et ils ont été décrits par les analyses des interactions verbales dans tous les types d'interactions et chez de nombreux locuteurs. Ce qui est spécifique avec les personnes en insécurité langagière c'est la fréquence de ces traits et leur accumulation au cours de la même interaction.

\section{Conclusion : de l'insécurité à la sécurisation en formation d'adultes}

Le concept d'insécurité langagière nous permet, non d'édulcorer la réalité, mais de rendre compte de façon plus fine des difficultés de certains adultes à interagir de façon efficace à l'écrit et à l'oral. La notion de «maitrise de la langue » a le double désavantage de mettre l'accent sur le code et de laisser penser qu'il existerait un stade d'appropriation repérable, une ligne de démarcation au-delà de laquelle 
les locuteurs et/ou les lecteurs-scripteurs se trouveraient en situation de dominer le code et son utilisation. Par ailleurs, le concept de sécurisation langagière, au contraire, indique un continuum, un processus d'appropriation, dont les étapes ne sont pas prédéfinies. Nous avons forgé ce concept en partant du terrain de la formation des adultes et non sur la base d'une approche théorique que nous aurions tenté de faire coller à la réalité. Le processus de sécurisation langagière est un parcours de formation. C'est celui que suivent les adultes engagés dans des dispositifs d'insertion et d'intégration avec leurs volets linguistiques. C'est sur l'analyse des pratiques, des contenus et des objectifs de ces formations que nos recherches en didactique se concentrent.

Les adultes qui nous intéressent dans cet article appartiennent très majoritairement aux catégories populaires et sont engagés dans des dispositifs de formation, d'intégration et de formation linguistique pour les migrants ou d'insertion sociale et professionnelle. Certains dispositifs de formation sont spécifiques aux migrants et certains le sont aux francophones mais d'autres accueillent des personnes de toutes origines linguistiques. L'objectif ultime de ces dispositifs de formation est le plus souvent l'emploi, qui est la clé de tout processus d'intégration et d'insertion. Qu'il s'agisse de trouver un emploi, de le conserver, de s'adapter à un nouveau poste ou de se reconvertir, le travail est le pôle magnétique de presque toutes les actions de formation linguistique. Or, la «part langagière du travail » ne cessant de gagner du terrain (Boutet 2001), y compris dans les métiers traditionnellement réservés aux salariés les moins dotés en capital scolaire (métiers de la propreté, BTP, hôtellerie, etc.), l'insécurité langagière des travailleurs en poste ou en recherche d'emploi devient un obstacle de moins en moins « contournable».

C'est pourquoi nous pensons nécessaire de faire converger des domaines de recherche qui, chacun à leur manière mais jusqu'ici de façon dispersée, travaillent sur cette question: sociolinguistique, didactique des langues, didactique professionnelle, ergonomie, etc. C'est 
l'objectif du réseau Langage, Travail et Formation (LTF) que nous avons créé.

\section{Bibliographie}

Adami, H. (2008). Tests de sécurité pour salariés en insécurité à l'écrit. TransFormations, $\mathrm{n}^{\circ} 1$.

Bernstein, B. (1975). Langage et classes sociales: codes sociolinguistiques et contrôle social. Paris, Les Editions de Minuit.

Adami, H., André, V. (à paraître). Corpus et apprentissage du FLI. Lynx. André, V. (2009). Les compétences langagières des métiers de la propreté: de l'analyse des situations de communication à la formation professionnelle. Bulletin suisse de linguistique appliquée, 90, 149-165.

Alber, J.L., Py, B., 1985, Interlangue et conversation exolingue, Cahiers du département des langues et sciences du langage. 1, Lausanne Université, 30-47.

Alber, J.L., Py, B., 1986, Vers un modèle exolingue de la communication interculturelle: interparole, coopération et conversation. Etudes de linguistique appliquée, $\mathrm{n}^{\circ} 61,78-90$.

Besse, J.M., 1995, L'écrit, l'école et l'illettrisme. Paris, Magnard.

Bourdieu, P. (1980). La distinction. Critique sociale du jugement. Paris, Editions de minuit.

Bourdieu, P. (1982). Ce que parler veut dire. L'économie des échanges linguistiques. Paris, Fayard.

Bourdieu, P. (Dir.). (1993). La misère du monde. Paris, Editions du Seuil. Boutet, J. (2001). La part langagière du travail. Langage et société, 98, 17-42.

Canut, E. et Vertalier, M. (2008). L'apprentissage du langage, une approche interactionnelle. Paris, L'Harmattan.

De Certeau, M., (1990) (Réed). L'invention du quotidien. Tome 1 : Arts de faire. Gallimard, Paris.

De Certeau M., Julia D. et Revel J. (2002). Une politique de la langue. Paris, Folio. 
De Pietro (1988). Conversations exolingues. Une approche linguistique des interactions culturelles. In Cosnier, J., Gelas, N., Kerbrat-Orecchioni, C., (éds), Décrire la conversation. Paris, Editions du CNRS.

Ferreiro, E. (2001). Culture écrite et éducation. Paris, Retz.

Goody, J. (1979). La raison graphique: la domestication de la pensée sauvage. Paris, Editions de Minuit.

Goody, J. (1986) La logique de l'écriture: aux origines des sociétés humaines. Paris, Armand Colin.

Goody, J. (1994). Entre l'oralité et l'écriture. Paris, Presses Universitaires de France.

Goody, J. (2007). Pouvoirs et savoirs de l'écrit. Paris, La Dispute.

Grignon, C et Passeron, J.C. (1989). Le savant et le populaire. Misérabilisme et populisme en sociologie et en littérature. Paris, Gallimard Le Seuil.

Hoggart, R. (1970). La culture du pauvre. Paris, Les Editions de Minuit. Hymes, D. (1972). Models of the interaction of language and social life. In Gumperz, J. and

Hymes, D. (Eds), Directions in Sociolinguistics. The Ethnography of Communication. Oxford, Basil Blackwell, 35-71.

Hymes, D. (1984). Vers la compétence de communication. Paris, Hatier, Collection Langues et apprentissage des langues.

Labov, W. (1976). Sociolinguistique, Paris. Editions de minuit.

Lahire, B. (1993a). La raison des plus faibles. Rapports au travail, écritures domestiques et lecture en milieux populaires. Lille, Presses universitaires de Lyon.

Lahire B. (1993b). Culture écrite et inégalités scolaires. Lyon, Presses universitaires de Lyon.

Lahire, B. (1998). Tableaux de famille. Heurs et malheurs scolaires en milieux populaires. Paris, Gallimard/Seuil, « Hautes Etudes ».

Lahire, B. (2005). L'invention de l'illettrisme. Paris, La Découverte. Langbach, V. (2014). Analyse et mesure de l'insécurité langagière chez des adultes francophones natifs en insertion. Thèse de doctorat. Université de Lorraine. 
Lapoutre D. (2001). Cour de banlieue. Codes, rites et langages. Paris: Odile Jacob.

Leclercq, V. (1999) Face à l'illettrisme: enseigner l'écrit à des adultes. Paris, ESF Editeur.

Leclercq, V. (2007). La formation de base: publics, dispositifs, pratiques. Savoirs, n¹4, 8-55.

LTF (2012). Perceptions et définitions des salariés en insécurité langagière. Séminaire du Réseau Langage, Travail et Formation. Nancy, le 29 juin. https://apps.atilf.fr/reseaultf/?p=166

Moirand, S. (1982). Enseigner à communiquer en langue étrangère. Paris, Hachette.

Sansot, P. (1991). Les gens de peu. Paris, Presses Universitaires de France.

Schwartz, 0. (2011). Peut-on parler des classes populaires? Laviedesidees.fr, http://www.laviedesidees.fr/IMG/pdf/20110913 schwartz.pdf Verret, M. (1996). La culture ouvrière, Paris, L'Harmattan.

Contact: Herve.Adami@univ-lorraine.fr et Virginie.Andre@univlorraine.fr 